\title{
A CONTINUOUS TRANSFORMATION USEFUL FOR DISTRICTING
}

\author{
W. R. Tobler \\ Depariment of Geography \\ University of Michigan \\ Ann Arbor, Michigan 48104
}

Suppose that one could stretch a geographical map so that areas containing many people would appear large, and areas containing few people would appear small. On a rubber map, for example, every person might be represented by an inked dot. We now jmagine the rubber sheet to be stretched so that all the dots are at an equal distance from each other (see Ruston ${ }^{1}$ ). If such a map could be constructed, then all perfect political districts should be the same size, for they should contain equal numbers of people. Alternately, one might wish to construct district boundaries by drawing them as hexagons on such a map. These general notions are made more precise and given mathematical definition in the paragraphs that follow. The political problem of distuicting is related to a classical theoretical problem in the field of geography. The location-theoretic problem of positioning service facilities (schools, hospitals, stores, cities, and so forth) in a geographic area of varying population density has a structure similar to the districting problem (see Bunge ${ }^{2}$ ). This theoretical problem in geography provided the main impetus for my research, and it is reflected in the results to be demonstrated.

Assume first that the relevant population density is described as a continuous nonnegative function of position, $h(x, y)$. A small rectangle bounded by $x, x+\Delta x$, $y, y+\Delta y$ then contains $h(x, y) \Delta x \Delta y$ people. This number is to be the same as the area of a small rectangle bounded by the lines $u, u+\Delta u, v, v+\Delta v$ on the final diagram. The condition equation thus becomes

$$
\mathrm{h}(\mathrm{x}, \mathrm{y}) \Delta \mathrm{x} \Delta \mathrm{y}=\Delta \mathrm{u} \Delta \mathrm{v},
$$

Passing to differentials, we have

$$
h(x, y) d x d y=d u d v,
$$

and, inserting the Jacobian determinant for a change of variables

$$
h(x, y) d x d y=\left(\frac{\partial u}{\partial x} \frac{\partial v}{\partial y}-\frac{\partial u}{\partial y} \frac{\partial v}{\partial x}\right) d x d y .
$$

The basic differential equation of the system is thus $\mathbf{J}=\mathbf{h}(\mathbf{x}, \mathrm{y})$. This is immediately seen to be a generalization of the notion of an equal area mapping and easily extended to a sphere or ellipsoid (see Tobler $^{3}$ ). We do not wish to place any restrictions, other than boundedness, on the density function $h$, thus ruling out some simpler solutions to this equation, as for example might be suggested if $h$ were separable, or centrally symmetric when given in polar coordinates. The basic differential equation defines an infinite number of possible distorted maps or cartograms. The necessary next step is to add further conditions, and the one which comes to mind immediately is rather obvious. The distorted map should look as much like the usual map as possible or-in keeping with the present context-should maintain compactness as nearly as possible. These two statements turn out to be mathe- 
matically equivalent and require that the transformation be as nearly conformal as possible. The entire problem is thus given as

$$
\begin{aligned}
& \text { minimize } \iint_{R}\left[\frac{\partial \mathrm{u}^{2}}{\partial \mathrm{x}}+\frac{\partial \mathrm{u}^{2}}{\partial \mathrm{y}}+\frac{\partial \mathrm{v}^{2}}{\partial \mathrm{x}}+\frac{\partial \mathrm{v}^{2}}{\partial \mathrm{y}}\right] \mathrm{dx} \mathrm{dy} \\
& \text { subject to } \frac{\partial \mathrm{u}}{\partial \mathrm{x}} \frac{\partial \mathrm{v}}{\partial \mathrm{y}}-\frac{\partial \mathrm{u}}{\partial \mathrm{y}} \frac{\partial \mathrm{v}}{\partial \mathrm{x}}-\mathrm{h}(\mathrm{x}, \mathrm{y}) \equiv 0
\end{aligned}
$$

which requires only minor modification to be adapted to the terrestrial sphere or ellipsoid.

An analytical solution to this pair of equations is not known, but an iterative finite difference method has been programmed. The basic algorithm is as follows. Consider a rectangle on the map with bounding vertices $u_{i}, v_{i}, i=1,2,3,4$. The area of this rectangle is easily computed as a function of the values of vertex coordinates and can be compared with the desired area as defined by $h$, suitably normalized. By adding small increments $\Delta u_{i}, \Delta v_{i}, i=1,2,3,4$, to each of the vertex coordinates we can change the area of the rectangle. It is only necessary to compute the appropriate values for $\Delta \mathrm{u}_{\mathrm{i}}, \Delta \mathrm{v}_{\mathrm{i}}, \mathrm{i}=1,2,3,4$. These values are obtained by writing the equation for the difference in area as a function of the modifications to the vertices. But there are eight values to be found, and only one equation. We now invoke the near conformality requirement which says that the change is to be a similitude. The single increment to the vertex coordinates is now found to be given by a quadratic function involving the difference between the desired and actual area of the rectangle.

This completes the adjustment for one rectangle. An interior vertex point, however, will be connected to four rectangles, and the latter may conflict in their desired adjustments. The vector mean of the displacements is used in this case, and the program cycles back for another iteration. The method is easily extended to cells of arbitrary shape. The program converges but it is slow, because before any vertex is adjusted a topological test must be performed to keep vertices from crossing the boundary of any cell, and boundaries are not allowed to cross over vertices. A mean square error criterion has been adopted to measure the efficacy of the iterations.

The United States has been used as a first example, beginning with an estimate of the number of people contained within each one degree quadrilateral of latitude and longitude. The computations start from an initial configuration in which the cell vertices are defined by integral values of latitude $(\varphi)$ and longitude $(\lambda)$, with a small adjustment for the mean latitude. The iterations produce a table of $u, v$ coordinates for the bounding vertices of each cell; i.e., we have a one-to-one mapping

$$
(\varphi, \lambda) \rightarrow(u, v)
$$

Using double bivariate interpolation, a map projection plotting program, and a magnetic tape containing an outline of the United States, a complete cartogram can be plotted (see Figure 1). On this map we define a set of compact cells, hexagons, in $u, v$ coordinates. Another program computes the inversion

$$
(u, v) \rightarrow(\varphi, \lambda)
$$

and this allows us to map the hexagons back onto the regular United States map. Each cell now contains an equal number of people, and this result is exact to the extent that the iterations have converged. It is obviously not valid in the oceanic areas, where some population has been allocated for aesthetic reasons. 


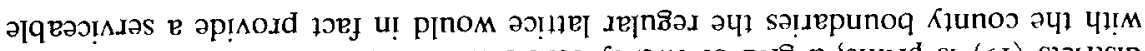

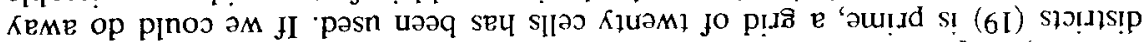

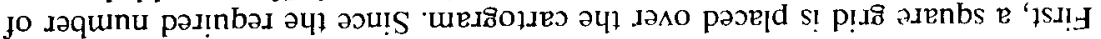

'suogiexay 8u!sn salełs pal!u

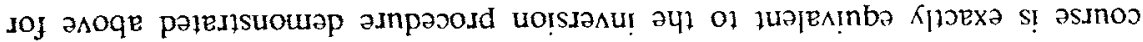

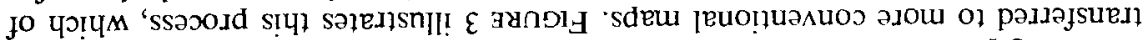

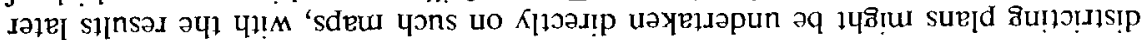

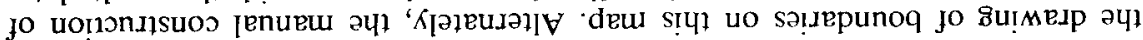
Кq КІІ

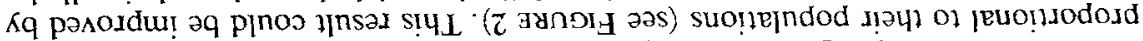

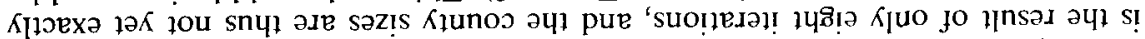

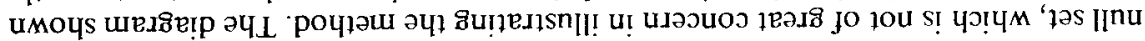

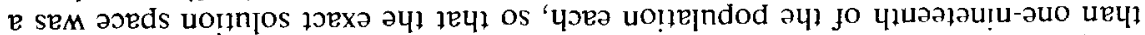

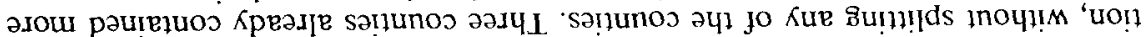

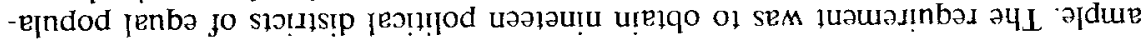

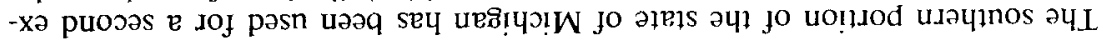

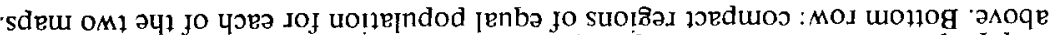

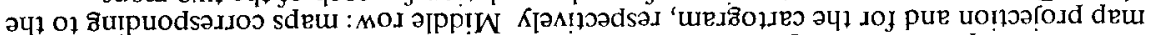

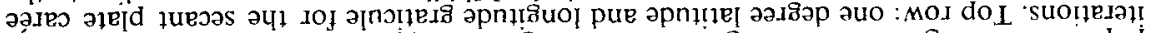

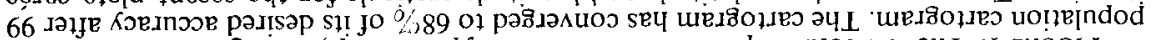

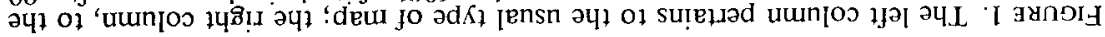
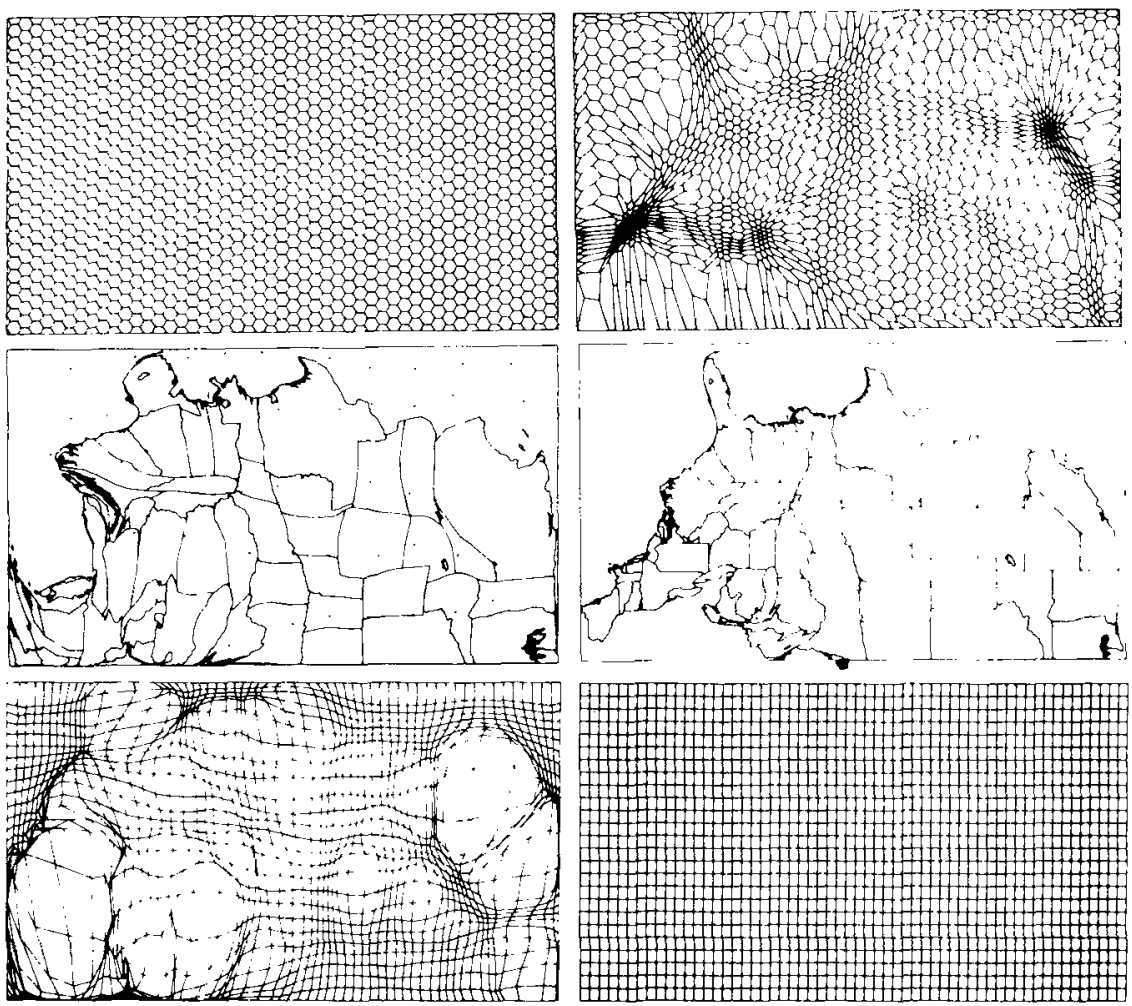

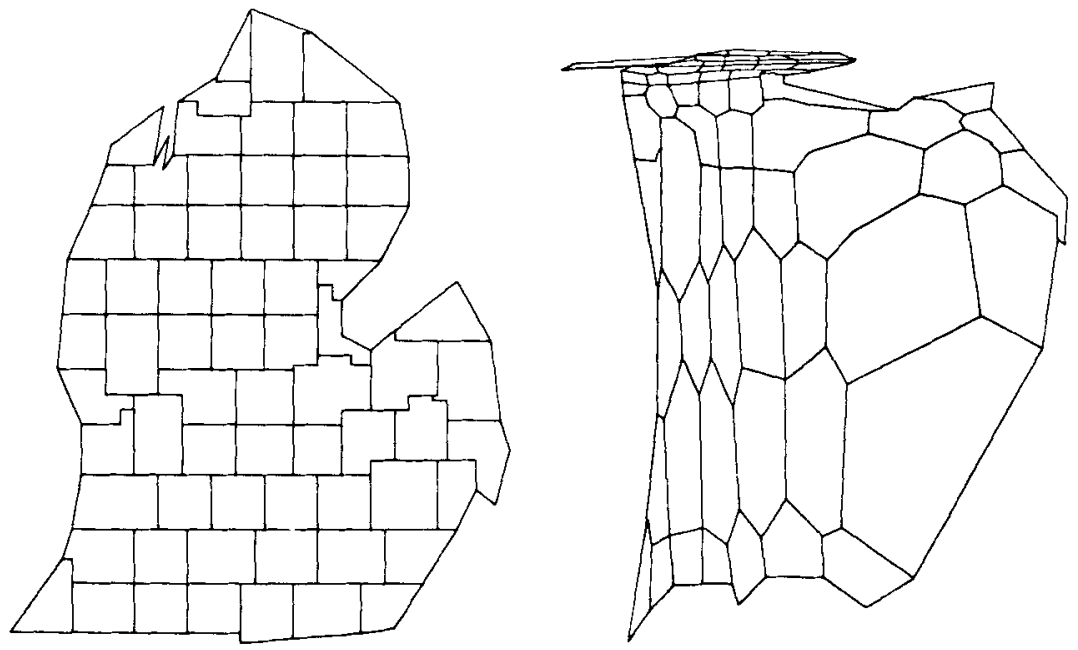

FIGURE 2. Southern Michigan: normal county map (left) and 1970 population cartogram after eight iterations.
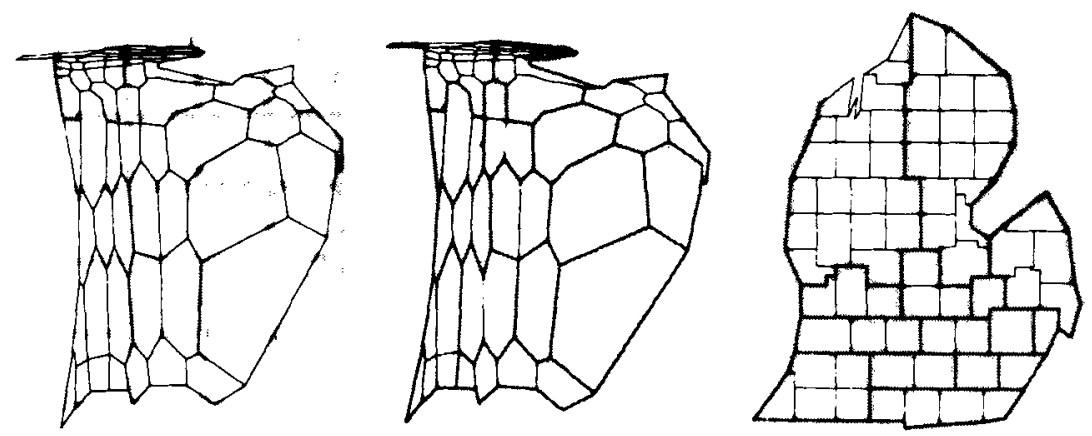

FigURE 3. Districting on a cartogram. From left to right: approximation by a regular grid, accommodation to the required boundaries, and transfer to the criginal map.

set of districts. Since we cannot, by the assumptions of the problem, get rid of the county boundaries, let us consider the grid as suggestive of what the final appearance of the districts should be. More precisely, let us attempt to find a set of districts the boundaries of which depart as little as possible from the grid. "As little as possible" can be taken in the least squares sense, meaning that some measure of mismatch, e.g. the area between the boundaries of the districts and the edges of the cells, must be minimized. A formal solution of the problem stated in this manner does not seem easier than the initial problem. Visually and heuristically we begin by attempting to match the edge of the grid with the edge of the map. (The map can be made to fit exactly into a rectangle of the proper proportions, but this has not been done in the example.) Now pick those county lines that fall near grid lines, and do a lot of fudging to obtain the requisite number of districts ( 15 in the present instancerecalling that one of the counties is already bigger than three optimal districts and 
that we do not allow splitting of counties, by our assumptions). Finally transfer the lines back to the original map. The mean percentage error of the districting on this first attempt is $44.6 \%$. This result is attrocious, even though most of the error must, at least in this instance, be attributed to the initial conditions of the problem.

As a third example, assume that the population density of a city can be described to an arbitrary degree of accuracy by a mathematical equation, and that the first term in such an expansion (see Tobler ${ }^{4}$ ) is

$$
\mathrm{D}=\mathrm{f}(\mathrm{r})=\frac{6 \mathrm{P}}{\pi \mathrm{R}^{4}}(\mathrm{r}-\mathrm{R})^{2}
$$

where

$$
\begin{aligned}
\mathbf{D} & =\text { number of persons per square kilometer } \\
\mathbf{P} & =\text { total population } \\
r & =\text { distance in kilometers from the center of town } \\
\mathbf{R} & =0.035 \mathrm{P}^{0.44}, \text { the radius of the town } \\
\pi & =3.14159 \ldots
\end{aligned}
$$

In this "parabolic" town, densities do not vary with direction, so that a natural, not necessarily optimal, solution to the defining differential equation in polar coordinates is given by

$$
\begin{aligned}
& \mathbf{r}^{\prime}=\sqrt{\frac{\mathrm{P}}{\pi}\left(\frac{3 \mathrm{r}^{4}}{\mathrm{R}^{4}}-\frac{8 \mathrm{r}^{3}}{\mathrm{R}^{3}}+\frac{6 \mathrm{r}^{2}}{\mathrm{R}^{2}}\right)} \\
& \theta^{\prime}=\theta
\end{aligned}
$$

FIGURE 4 shows this transformation applied to a simple street map of the city of Ann Arbor, whose population is 100,000 . This population cartogram may be used as the previous maps were if one keeps in mind the several approximations that were invoked during its construction. The polar coordinate form of solution is especially advantageous when pie-shaped districts are specified by the legal system.

The foregoing three examples demonstrate application of a continuous transformation to a districting problem with data given for a cellular lattice, for irregular polygons, and by an approximating equation, at national, regional, and local
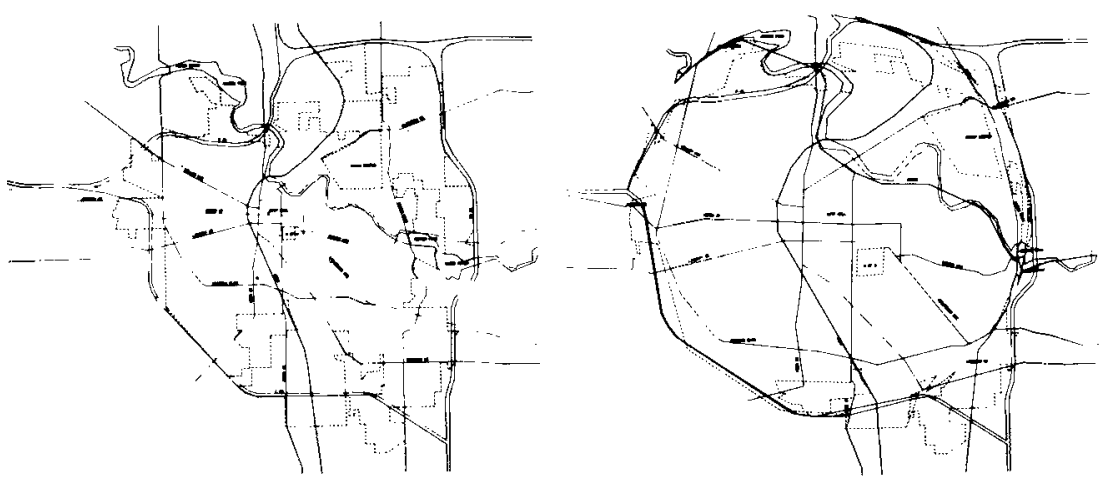

FigURE 4. Left: conventional map of Ann Arbor. Right: population cartogram of Ann Arbor. 
scales. A definite improvement in accuracy and especially in computational speed would be achieved if an analytical solution were available for the system of differential equations.

\section{REFERENCES}

1. Ruston, G. 1971. Map transformations of point patterns. Papers Regional Sci. Assoc. In press.

2. Bunge, W. 1966. Theoretical Geography. 2nd edit. Geerlups Lund, Sweden.

3. ToBler, W. 1963. Geographic area and map projections. Geog. Rev. 53(1): 59-78.

4. ToBler, W. 1969. The spectrum of U.S. 40. Papers of the Regional Science Assoc. 23: $45-52$. 